\title{
Parallel Implementation of a Video-based Vehicle Speed Measurement System for Municipal Roadways
}

\author{
Abdorreza Joe Afshany \\ Department of Computer Engineering, University of Guilan, Rasht, Iran \\ E-mail: joe_afshany@msc.guilan.ac.ir \\ Ali Tourani ${ }^{1}$, Asadollah Shahbahrami ${ }^{1}$, Saeed Khazaee ${ }^{2}$, and Alireza Akoushideh ${ }^{3}$ \\ ${ }^{1}$ Department of Computer Engineering, University of Guilan, Rasht, Iran \\ ${ }^{2}$ Centre for Pattern Recognition and Machine Intelligence, Concordia University Montreal, Canada \\ ${ }^{3}$ Shahid-Chamran College, Technical and Vocational University, Tehran, Iran \\ E-mails tourani@msc.guilan.ac.ir, shahbahrami@guilan.ac.ir, s_khaza@encs.concordia.ca, akushide@sbu.ac.ir
}

Received: 21 April 2019; Accepted: 07 June 2019; Published: 08 November 2019

\begin{abstract}
Nowadays, Intelligent Transportation Systems (ITS) are known as powerful solutions for handling traffic-related issues. ITS are used in various applications such as traffic signal control, vehicle counting, and automatic license plate detection. In the special case, video cameras are applied in ITS which can provide useful information after processing their outputs, known as Video-based Intelligent Transportation Systems (VITS). Among various applications of V-ITS, automatic vehicle speed measurement is a fast-growing field due to its numerous benefits. In this regard, visual appearancebased methods are common types of video-based speed measurement approaches which suffer from a computationally intensive performance. These methods repeatedly search for special visual features of vehicles, like the license plate, in consecutive frames. In this paper, a parallelized version of an appearance-based speed measurement method is presented which is real-time and requires lower computational costs. To acquire this, datalevel parallelism was applied on three computationally intensive modules of the method with low dependencies using NVidia's CUDA platform. The parallelization process was performed by the distribution of the method's constituent modules on multiple processing elements, which resulted in better throughputs and massively parallelism. Experimental results have shown that the CUDA-enabled implementation runs about 1.81 times faster than the main sequential approach to calculate each vehicle's speed. In addition, the parallelized kernels of the mentioned modules provide $21.28,408.71$ and 188.87 speed-up in singularly execution. The reason for performing these experiments was to clarify the vital role of computational cost in developing video-based speed measurement systems for real-time applications.
\end{abstract}

Index Terms-Parallelism, speed measurement, video processing, intelligent transportation systems.

\section{INTRODUCTION}

For several years, providing safe and secure transportation circumstances have been considered as the basic requirement for the development of industries and increasing social welfare level in developed countries [1]. Nowadays, transportation issues such as environmental pollutions, reduction of energy resources, corporeal and financial damages caused by car accidents and the rapid growth trend of transportation demands - especially during the peak hours of road traffics - have become an unbreakable challenge in all cities around the world [2]. In this regard, Intelligent Transportation Systems (ITS) are defined as the means for collection of tools, facilities, and specializations, such as traffic management and telecommunications technologies in the form of coordinated instruments. ITS have various branches to provide the desired solutions to tackle the mentioned issues [3]. One of the main applications of ITS is the automatic speed measurement. Due to the possible dangers such as vehicle-pedestrians' accidents, vehicles' speed control procedure on urban roadways is very important. There are several methods for speed measurement purposes and many systems designed over the time to calculate the passing vehicles' speeds, like inductive loop detectors, Laser-based (Lidar) and Radarbased systems [4]. Inductive loop detectors are known as widely used instruments, but they suffer from some major problems such as challenging installation and maintenance, short lifetime and road damage [5]. On the other hand, Laser-based and Radar-based systems are more expensive than inductive loop detectors, but they have the advantage of better accuracy [6]. Here, the accuracy is defined as the proximity of a vehicle's real 
instantaneous speed and calculated speed. As another type of devices, speed sensors using Digital Image Processing (DIP) have attracted huge interest among researchers in recent years. These systems, which known as vision-based approaches, use the video output of installed road cameras and process them to obtain information about the vehicle's speed [7]. Vision-based methods can be remarked as alternatives for existing speed measurement systems, i.e. Radar and Laser-based applications, in case they provide acceptable accuracy. Although these approaches suffer from some limitations like high computational cost and some challenges in detecting and tracking vehicles in the video scene, measuring vehicle speeds using DIP has several advantages such as lower cost, easier maintenance, and better expandability.

In this paper, we introduce a parallel implementation of a formerly presented computationally intensive visionbased vehicle speed measurement method [12] to provide a real-time performance by utilizing GPU for parallelization. Experimental results have shown about $55.12 \%$ decrease in the execution of the parallelized version compared to the CPU-based approach. It should be noted that some minor changes in vehicle detection and tracking modules have been applied which are thoroughly explained in related sections. The main contributions of our work are summarized below:

1) We developed a parallel implementation of a sequential (CPU-based) speed measurement approach;

2) General analysis and profiling of the method to detect computationally intensive modules with low dependencies to other modules was performed;

3) The effect of parallelization in both kernel and application levels was calculated;

4) We observed that by parallelizing some computationally intensive modules made the method robust against executing in almost realtime applications;

The rest of the paper is organized as follows: we will first explain the definitions and some related works in Section 2. In Section 3, the description of the proposed method which is a parallelized implementation of a speed measurement approach including motion detection, license plate recognition, vehicle tracking, and speed calculation modules is introduced. Experimental results and evaluations are presented in Section 4 and finally, we finish with conclusions in Section 5.

\section{RELATED WORKS}

In this section, some primary concepts are introduced to provide a better understanding of the speed measurement process and later, some related works are presented and discussed. As a common classification of vision-based speed measurement approaches, two main categories, including motion-based and appearance-based methods are existed [7]. Motion-based approaches do not depend on visual features of the vehicles and instead, require a sequence of frames to detect moving vehicles. Although these methods are able to recognize the depth of the scene, they do not represent vehicles by their visual features and thus, they provide lower computational costs. Appearance-based approaches, on the other hand, need some visual features of the vehicles, e.g. license plate or tail-light, in each frame. As a common manner, vehicle speed measurement algorithms using DIP have a general block diagram as shown in Fig. 1. They come along with some differences in applying algorithms for each part of the scenario which may result in different computational costs and performance. As it can be mentioned, in the first step, the general topographies of moving vehicles in the scene should be detected using various methods such as background subtraction or frame differencing in motion-based, and visual pattern or texture in appearance-based approaches. These features may be existed in the whole or some special regions of vehicles, like the headlights, license plate, etc. In the next step, the previously found features should be tracked among sequential frames to provide the vehicle's displacement in pixels. Tracking process makes it possible to measure the amount of the moving vehicle's displacement to provide speed measurement parameters. The final results of this process can be some special parts of the vehicles or the whole vehicle's shape. Finally, a module to calculate vehicle speed through mapping pixels to meters and frame numbers to seconds is performed. This mapping function should convert the displacement vector $\overrightarrow{d_{l}}$ in the camera's focal length to the displacement vector $\overrightarrow{D_{l}}$ in real-world metrics.

\begin{tabular}{|c|c|c|c|}
\hline $\begin{array}{c}\text { Detecting Moving } \\
\text { Vehicles }\end{array}$ & $\begin{array}{c}\text { Features } \\
\text { Extraction } \\
\text { \& Selection }\end{array}$ & $\begin{array}{l}\text { Features } \\
\text { Tracking }\end{array}$ & $\begin{array}{c}\text { Speed } \\
\text { Measurement }\end{array}$ \\
\hline
\end{tabular}

Fig.1. A general block diagram of common vision-based speed measurement methods.

Because of the direct recognition of vehicles in separate frames, these methods are faced with high computational cost and time consumption to provide appropriate accuracy. In this regard, parallelization of such algorithms is an appropriate process to tackle high computational costs by concurrent execution of procedure divisions on various processing units. In recent years, Graphical Processing Units (GPUs) have become significantly powerful tools for parallelization purposes. GPUs, with the help of their abundant number of processing units, are able to provide SIMT (Single Instruction Multiple Threads) parallelization and execute a method in a fraction of time required for the execution of the same method on CPUs.

\section{A. Background Information}

In this sub-section, some important definition of concepts and background information which have been used in the upcoming parts of the paper are presented [811]:

- $\quad$ Compute Unified Device Architecture (CUDA): 
in order to utilize a Graphical Processing Unit as a powerful device to speed up computationally intensive algorithms, CUDA has been developed by NVidia as a great programming tool for parallelization. Before introducing CUDA, the task of GPU programming was tough and the programmer needed to know the main architecture of the GPU. CUDA simplified the implementation of GPU-enabled applications to be rendered on NVidia GPUs. Computationally intensive applications with the lowest possible dependencies are the best candidates for parallelization on CUDA. This environment makes programmers able to distribute the amount of computation in their codes on thousands of cores of their GPU cards and consequently, provide the performance equal to tens of CPUs with much less cost. In CUDA, kernels are referred to data-parallel portions of an application, which contain several threads for parallel execution to be operated on data stored in the GPU's memory. It should be mentioned that the process of initiating the kernels is done by CPU. For parallelization of an application, these threads should be grouped together to provide warps and blocks of codes. The main challenge for the programmer is to avoid serially execution of threads and provide optimized performance.

- Good features: Since selecting appropriate features equivalent to the physical points in ground truth is a difficult process, correct detection of these features is so important in object tracking goals. A "good feature" as it is mentioned in [9], is a region with high-intensity variations in more than one direction, like the areas of texture or corners. In this regard, Good Features to Track [9] is a corner detection approach based on the Harris corner detector which finds the strongest corners in an image and skips the corners below a pre-defined quality. So the output of this function is a number of corners which are appropriate for later tracking that makes the system needless of extracting information from every single corner in an image.

- Motion History Image (MHI): MHI is a common vision-based method for detecting moving objects in sequential frames which uses a static image template to understand the location and path of the motion. This technique has some advantages such as insensitivity to silhouette noises, holes, shadows and missing parts, and the ability of implementation in low illumination conditions. In MHI method, the intensity of each pixel in a temporal manner is used for motion representation, and a history of changes at each pixel location is stored for motion detection purposes.

- Pyramid version of Kanade-Lucas-Tomasi
$(K L T)$ : because the traditional algorithm of KLT only works for small displacements (in the order of one pixel), the pyramid version of this method is used in [10] to overcome the limitation of larger displacement detections. The pyramid version of KLT algorithm picks up a pyramid for each frame, where the image with the main dimensions is placed at the base of the pyramid. In each level, the width and height of the image are reduced by half. The pyramid KLT algorithm begins to find the vector $\vec{d}$ of displacement from the last level of the pyramid and uses the results for the initial estimation of $\vec{d}$ at the next level. This process continues to reach the base of the pyramid, i.e. the original image.

- T-HOG text descriptor: this text descriptor which was first presented in [8], detects a collection of characters by obtaining a gradient histogram of the top, middle and bottom of an image area by the means of the histograms of text regions. These areas have significant and fundamental differences with other non-text regions. Consequently, this method can be used to detect a vehicle's license plate regions in a video frame for further processes.

\section{B. Related Works}

Due to the numerous benefits of video-based ITS approaches, some different methods for estimating and measuring the speed of vehicles on the roadways are proposed. Most of these techniques use background/foreground segmentation algorithms to detect vehicles and track them in sequential frames to calculate their displacement in a period of time. These approaches follow the steps shown in Fig. 1 in most of the cases. In [12], a frame differencing technique to detect moving vehicles is presented that seeks a vehicle's license plate to extract desired features and track its good features in multiple frames using the pyramid KLT algorithm. The speed measurement average error in this approach was $-0.5 \mathrm{~km} / \mathrm{h}$ and in over $96.0 \%$ of cases, measurement errors were inside $[-3,+2] \mathrm{km} / \mathrm{h}$ range. Similarly, a robust approach presented in [13] used the same vehicle detection technique which considers each vehicle as a blob by the means of the edge detection method and tracks their centroids to calculate the displacements of blobs in a limited time range and measure the vehicles' speed. In [14], another frame differencing method to detect moving vehicles is presented which detects corners of the vehicle by Harris algorithm and tracks the centroid points using the Kanade-Lucas-Tomasi (KLT) method among sequential frames. After the tracking step, the vehicle's speed is calculated using a spherical projection that relates image movement with the vehicle's displacement. Authors in [15] used a frame differencing method for vehicle detection, which selects special points with large spatial gradients in two orthogonal directions within the vehicle's coverage area to track features. Therefore, the vehicle's speed was calculated by the means of velocity 
vectors obtained from the tracking step using an optical flow method. Similarly, in [16] a frame differencing and blob tracking method is presented in which the vehicle's speed is obtained by estimating each blob's displacement using static parameters. In other approaches, authors of [17-19] used a median filter for moving vehicle detection and calculated the real position of the vehicle in video frames to measure speed. A Gaussian distribution for detecting moving vehicles is presented in [20] which uses blob detection and tracking for speed calculation. In [21], the authors used the background/foreground segmentation technique to detect moving vehicles and blob tracking method to estimate their speed. Some other approaches like [22-24] used vehicles' license plates for detection purposes and by tracking the extracted features from the license plates, their speed was measured. In [22] detected characters using an Optical Character Recognition (OCR) algorithm which is inconstant in position and size are used for vehicle detection. This method requires a robust OCR and does not provide acceptable results even in a controlled environment. Similar work in [23] is done based on vehicles' license plate detection and tracking.

Although some of the mentioned methods are similar in detection or tracking steps, they have fundamental differences due to utilizing a wide variety of algorithms. Methods based on blob analysis, i.e. [13-14, 16, 18] and [34-35], are sensitive to environmental conditions such as shadow, perspective effect, and lighting changes. In addition, these methods only provide satisfactory results when the camera is fixed on top of the roadway. They are also computationally intensive due to their appearancebased methodologies. Some other methods used the same types of features from the blobs, such as [24] that detects the edges close to the boundaries of each blob, or [26] that extracts features including derivatives, Laplacian and colors from each blob. In [27], blob analysis problems were solved by direct tracking of unique features using the Lucas-Kanade optical flow algorithm, but according to the assumptions, this method could only track one vehicle in any timestamp [28].

Inspired by the mentioned approaches for vehicle detection and speed measurement, in this article, we implemented a parallel version of the vehicle speed measurement approach presented in [12]. The main reason for choosing this method is its robust performance, containing computationally intensive modules and the high potential of parallelization according to the authors' claims. This method takes advantage of license plate text features but does not require the characters of the license plate to be accurately segmented by the OCR algorithm to detect and track vehicles. Instead, the whole text appeared inside the license plate zone is recognized and tracked. The overall process of this approach includes four sequential steps, as it is shown briefly in Fig.2.

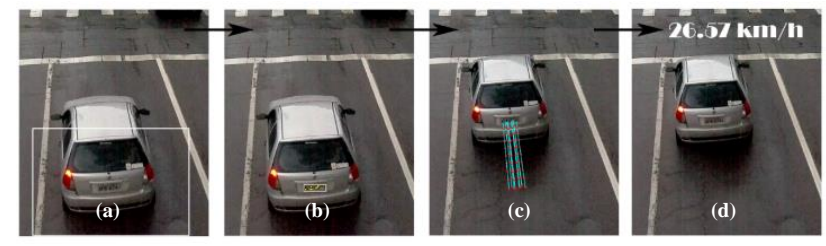

Fig.2. A block diagram of the speed measurement approach presented in [12]: a) Detecting a moving vehicle, b) Features extraction \& candidate selection, c) Features tracking, and d) Speed measurement.

The first step is to find the moving objects - i.e. vehicles - among consecutive frames in order to limit the whole process to a set of regions. This goal was reached using MHI motion detection algorithm presented in [29]. Then, the moving parts of the scene were considered as vehicles' boundaries and separated from the background scene. The frame differencing method produces a threshold binary image $D(x, y, t)$ where the pixels with value 1 in each frame are segmented and other remained pixels, use the maximum value of the same rate in the previous frame. This collection of the output pixels is stored as $H(x, y, t)$ in each frame which is shown in Equation (1), where $\tau$ refers to the duration of the motion in sequential frames.

$$
H(x . y . t)= \begin{cases}\tau & \text { if } D(x . y . t)=1 \\ \max (0 . H(x . y . t-1)-1) & o . w .\end{cases}
$$

Then, a binary segmentation mask $M(x, y, t)$ is acquired from $H(x, y, t)$ to collect the moving parts, where the values of $H$ larger than zero are presented as one in the mask. This process is presented in Equation (2):

$$
M(x . y . t)=\left\{\begin{array}{lr}
1 & \text { if } H(x . y . t)>0 \\
0 & o . w .
\end{array}\right.
$$

Formerly, by applying Vertical Projection Profile (VPP) [30], the left and right borders of vehicles are detected (considering the vehicles moving from the bottom to the top of the screen). VPP counts the number of pixels existed in each column of the mask $M(x, y, t)$ and stores the values in an array $V P$ with a length equal to the number of the mask's columns. After smoothing and normalizing $V P$, the exact range of pixels refers to the vehicle's presence in each frame are recognized and cropped by applying Find-Hills [12] method. By detecting the regions inside the vehicle's cropped area, the candidates of being the vehicle's license plate are extracted. Thus, some parts of the moving vehicle with a rectangular shape and white background are selected using Edge Extraction and Filtering method [31]. Furthermore, a module to merge neighboring edges remained after filtering is defined in which only the edges with a pre-defined size and ratio are selected using connected components labeling [32]. Among multiple 
candidates, using T-HOG [27] text descriptor which has been introduced in Section 2.1, the region with the most probability of being the vehicle's license plate will be extracted. The T-HOG descriptor will be used as an input for a Support Vector Machine (SVM) classifier and the output of the classifier shows whether the region belongs to a text or non-text area. In the next step and by detecting the license plate's region, a set of unique features inside the region is chosen for tracking. This process is done by good features extraction. The good feature, in this case, is a high-intensity region with some black pixels inside the white region, representing a vehicle's license plate. This feature is then tracked by the pyramid KLT tracking method introduced in Section 2.1 to provide the motion vector of the moving vehicle. In addition, a timer triggers as the vehicle enters the region of interest and stops as it leaves the region. Finally, each vector can represent the instantaneous speed of the vehicle at a specified time in pixels-per-frame metrics. Thus, a mapping function to convert it to the kilometersper-hour unit is necessary to be applied. As it has been proved in the pinhole camera model, for a single view of the scene, the homograph matrix $H M$ [33] can perform this mapping. Thus, according to the Equation (3), a plane-to-plane projective transformation and inverse perspective mapping can provide the final world plane metrics [37], where for a $3 \times 3$ homograph matrix $H M$, point $p_{i}\left(x_{i}, y_{i}\right)$ can be mapped to the point $\widehat{p_{w}}\left(x_{w}, y_{w}\right)$ in the world plane [36]:

$$
\left[\begin{array}{c}
x_{w} \\
y_{w} \\
1
\end{array}\right]=\left[\begin{array}{c}
z x_{w} \\
z y_{w} \\
z
\end{array}\right]=H M\left[\begin{array}{c}
x_{i} \\
y_{i} \\
1
\end{array}\right]
$$

$H M$ can be obtained from four points in the image with known coordinates in the real-world plane in the calibration step. These features are utilized to present the relocation of the vehicle and calculate its speed by mapping pixels-to-meters and frames-to-seconds.

As it has been acknowledged in the paper, the process of calculating $H(x, y, t)$ and $M(x, y, t)$ matrices is computationally intensive. To solve this issue, the author suggested to apply subsampling function [12], but it still would be a bottleneck for the performance of the system. We will discuss the structure of parallelization for better performance in Section 3.

\section{PROPOSED METHOD}

For parallelization, some time-consuming modules of the mentioned method were detected and implemented on GPU using CUDA programming environment. Although the mentioned approach is robust against high accuracy performance, it is considered as a time-consuming method due to containing multiple computationally intensive modules. In this section, the implementation of a CUDA-enabled version of the same method is presented in order to decrease the time required for execution. To investigate the effects of each module in the performance, we implemented the same approach
[12]. Fig.3 shows the time portion required for the execution of each module obtained by the means of profiling technique. To gather these time-shares, the implemented method was executed on several videos and the presented values are the average of the multiple calculated measures.

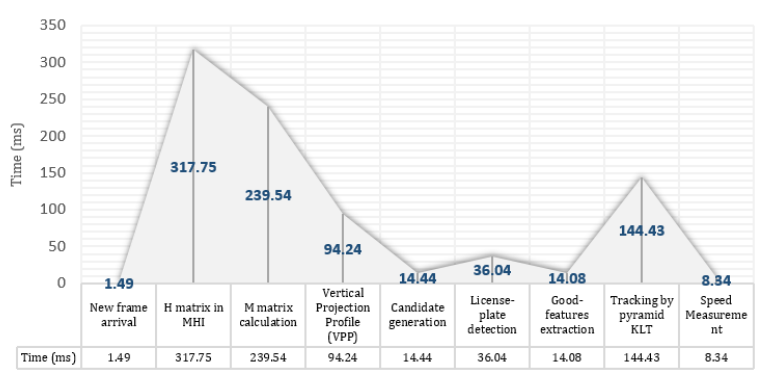

Fig.3. The time portion of various modules in the system (in milliseconds).

As it can be seen, the calculation of $H(x, y, t)$ and $M(x$, $y, t)$ matrices takes the most portion of time among the whole process, respectively. Vehicle tracking using the pyramid KLT is another computationally intensive module. In this regard, the system consumes totally 870.35 milliseconds to process the scene in order to detect and track vehicles in each frame; while the three mentioned modules consume $80.67 \%$ of the total time. The main reason of the huge time consumption in $H(x, y$, $t)$ and $M(x, y, t)$ matrices calculation is due to the system requirement for repeatedly performing the calculation for each pixel. These two modules have a rich data parallelism capability because they are made up of nested matrix multiplication operations. In addition, in the pyramid KLT tracking phase, the process of downsizing the vehicle's image and drawing motion vectors in each level of the pyramid needs a huge amount of calculations. According to Fig.3, VPP is another computationally intensive module which does not support running across multiple cores in parallel.

To optimize the performance of these three timeconsuming modules, we want to perform a parallelization by implementing their CUDA-enabled versions. In the parallel implementations, data-parallel portions of each module should be implemented as a CUDA kernel, where these kernels are manipulated by the main processor, i.e. CPU. Consequently, a $30 \times 30$ matrix of pixels was allocated as a block to the GPU to optimize the calculating process. A block is a group of threads referring to each pixel that should be processed and by aggregating them into Grids, the architecture of a parallel version of each module will be shaped. The parallelization logic should distribute light calculation workloads (such as matrix multiplication) of the selected modules on each thread with the lowest data fetching overhead and optimal memory allocation.

Fig. 4 shows the architecture of a GPU in brief [39]. According to Fig.4, there is a shared memory module and several registers inside each block which are allocated for calculations and shared for all threads of the block. In addition, each thread of a block has a unique local 
memory to store non-local variables, which are placed outside of the block. On the other hand, other memories including global, texture and constant memories are considered in GPU's architecture for higher efficiency and better control of processes and shared by all threads inside the Gird. Consequently, to provide a highefficiency system, it is necessary to utilize these memories and registers by the means of the CUDA platform.

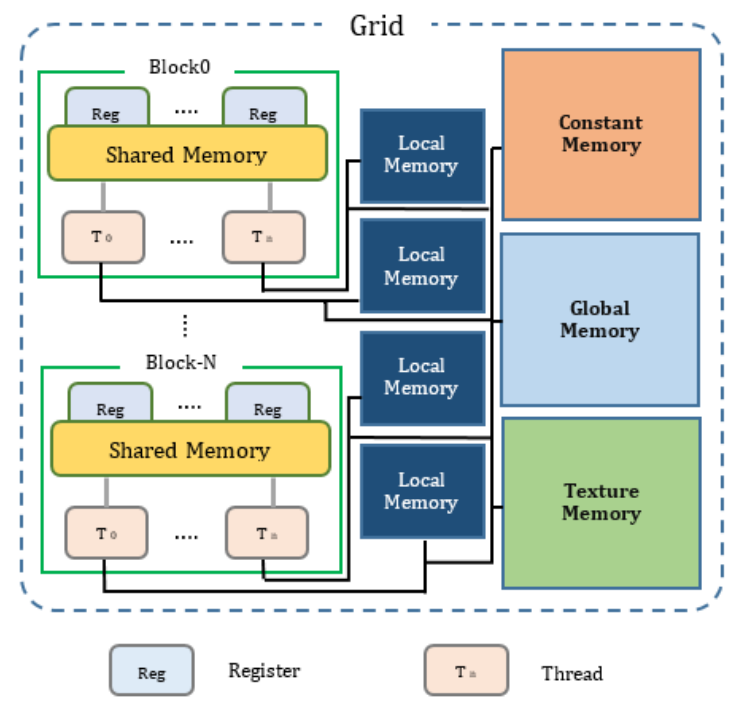

Fig.4. A common architecture of a GPU.

In order to parallelize $H(x, y, t), M(x, y, t)$ and pyramid KLT modules, we need to utilize GPU threads for each light-weight process. Fig.5 shows the CPU-based (sequential) version of MHI calculation according to Equation (1). As can be seen, all the pixels belong to the subtraction matrix of two sequential frames, named as diff should be checked as it was previously discussed in Equation (1). The current value of MHI module which is called $H$ in this figure is the output of the system based on the previous frame's MHI value prev. It should be noted that the variable mhi_duration is set to 5 in this approach, which means the system keeps tracking of five frames as the history to calculate the $H$ matrix.

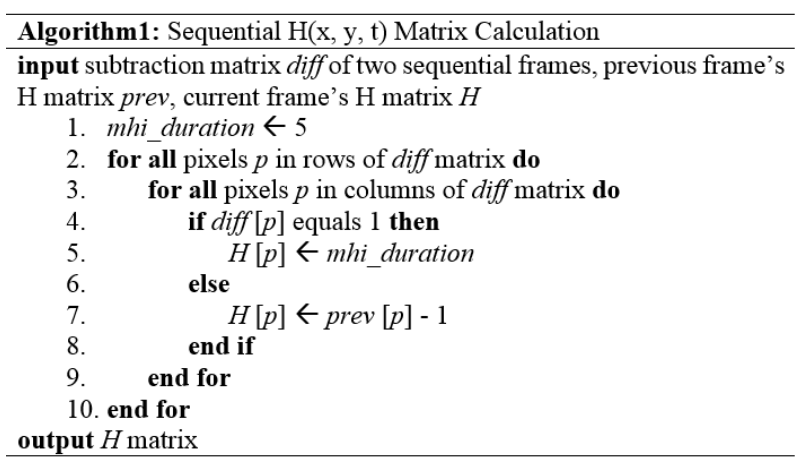

Fig.5. Sequential implementations of $\mathrm{H}(\mathrm{x}, \mathrm{y}, \mathrm{t})$ module.

In addition, Fig.6 shows the pseudo-code of the parallelized version of Algorithm1 presented in Fig.5. In this case, the block-size of the GPU is set to $30 \times 30$ which provides the best performance according to experiments and the resolution of the frames, named as $w$ and $h$ are utilized to calculate the grid based on block-size. In the parallel implementation, all the elements of the matrix diff are sequentially segmented into blocks and each block element should dedicate into a single processing thread. The pixels to thread mapping is done in $O(1)$ and the row-major fetching of the diff matrix element, makes the $H$ matrix calculation process to run in $O(w+h)$ instead of $O(w h)$.

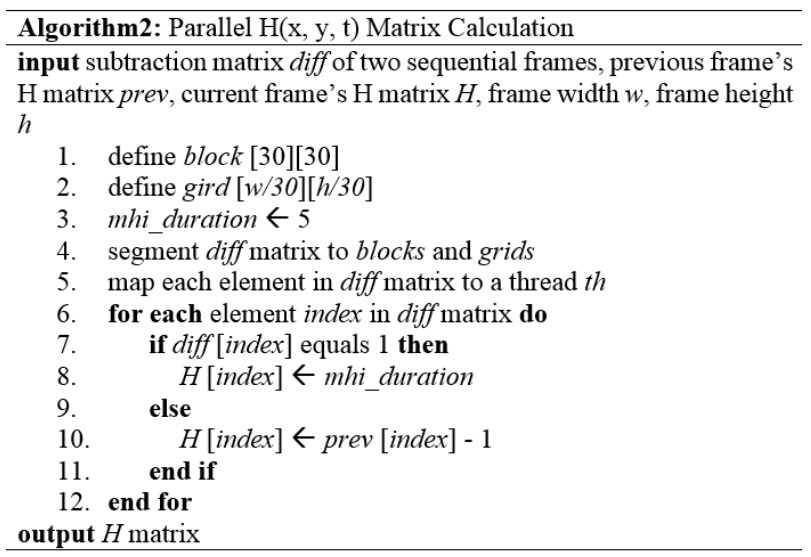

Fig.6. Parallel implementations of $\mathrm{H}(\mathrm{x}, \mathrm{y}, \mathrm{t})$ module.

Similarly, Fig.7 and Fig.8 show the CPU-based (sequential) and GPU-based (parallel) versions of $M(x, y$, $t)$ matrix calculation method. As it has been described before, $M(x, y, t)$ matrix is used for segmentation of $M H I$. Here, variable $M$ refers to the calculated mask $M(x, y, t)$ presented in Equation (2). The parallelization process of this module was the same as the method described in Fig. 6.

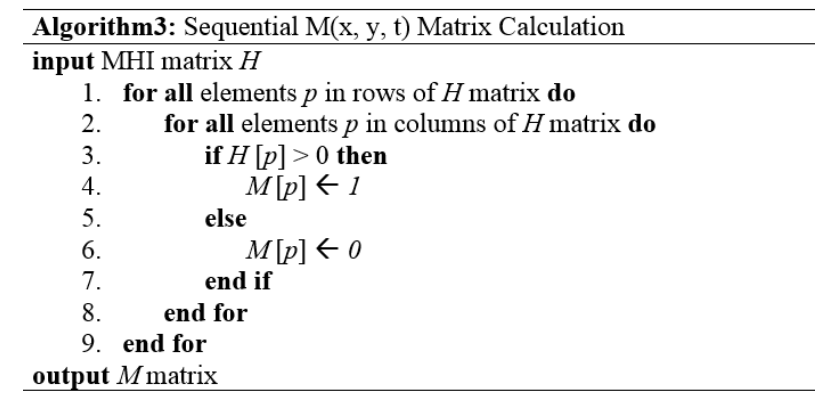

Fig.7. Sequential implementations of $\mathrm{M}(\mathrm{x}, \mathrm{y}, \mathrm{t})$ module.

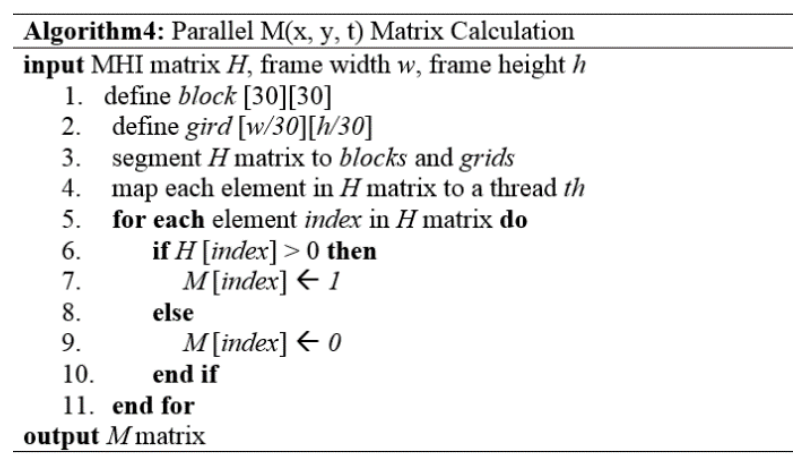

Fig.8. Parallel implementations of $\mathrm{M}(\mathrm{x}, \mathrm{y}, \mathrm{t})$ module. 
In addition, Fig.9 shows the output of the produced mask in the motion detection step. As it is shown, the moving vehicle in multiple frames is totally segmented from the background scene.

Finally, Fig.10 and Fig.11 illustrate the sequential and parallel versions of the pyramid KLT module, respectively. In these figures, previous and current frame matrices with the resolution of $w \times h$ are utilized to detect any relocation of the objects with special visual features and provide tracking of them in two sequential frames. These matrices are named prev_frame and curr_frame and the candidate features of being a license plate found by the good features algorithm are stored in prev_features and the curr_features arrays, respectively.
Firstly, the algorithm builds a pyramid with multiple levels, where the variable max_level defines the number of levels.

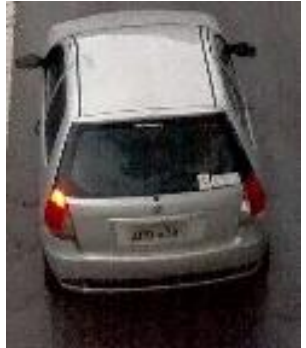

(a)

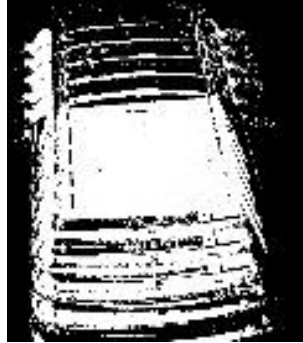

(b)
Fig.9. A sample frame (a), its binary segmentation mask M (b).

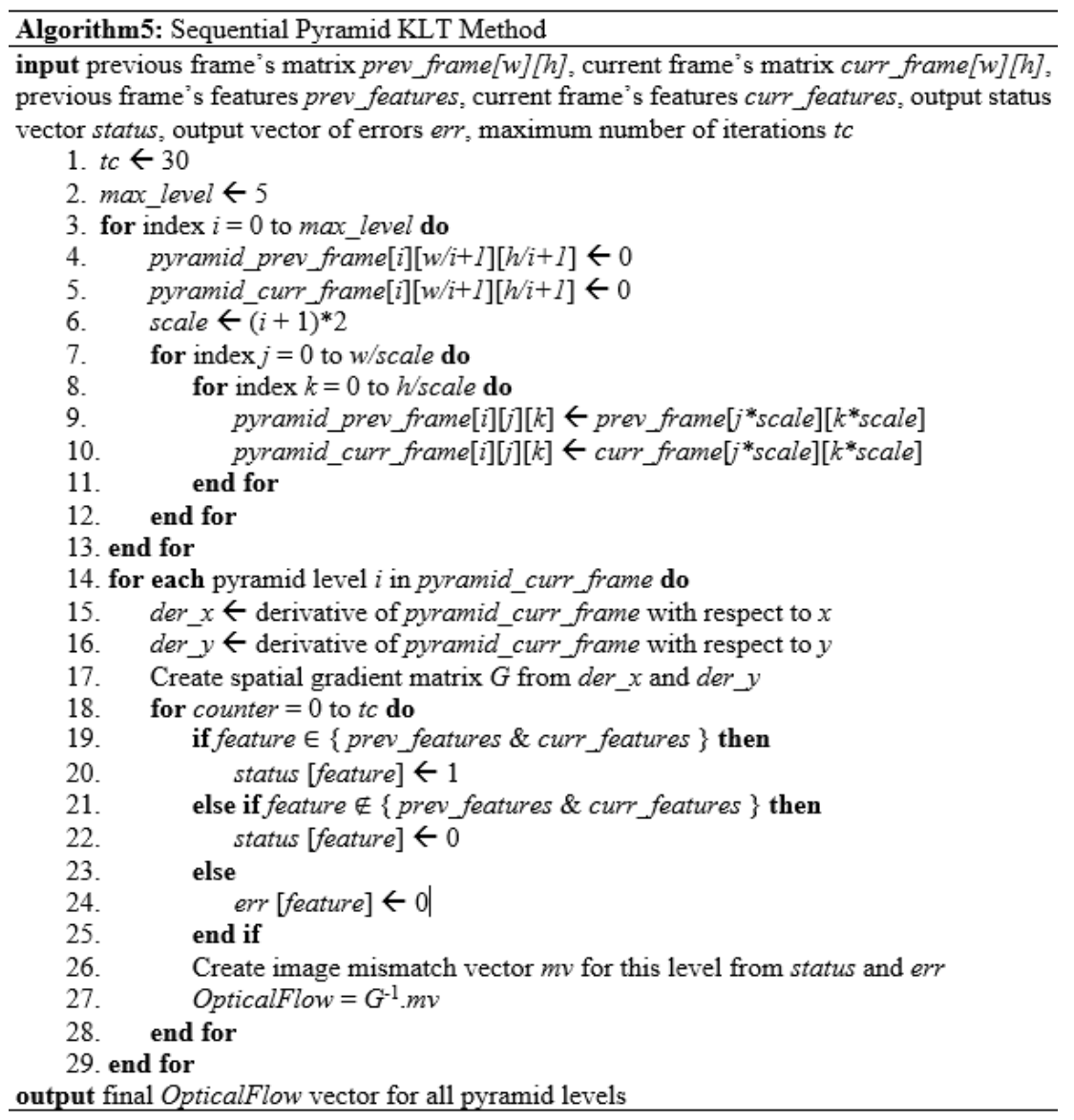

Fig.10. Sequential implementations of the pyramid KLT tracking module.

Each detected license plate from the detection step of the method lays in the base level of the pyramid and each higher level, stores the image of the license plate with half dimensions. Each pyramid version of the prev_frame and curr_frame frames are stored at pyramid_prev_frame $[i]$ and pyramid_curr_frame $[i]$ respectively, where $i$ is the corresponding level of the pyramid. After building the pyramid, if a feature found in both previous and current frames, its corresponding element in the status array becomes one and otherwise, it becomes zero. Similarly, the array err includes the type of errors occurred in tracking the corresponding feature. In addition, the variable tc stores the terminating conditions of the search module and the maximum number of pyramid levels is set to five by experiment in this approach. Finally, the OpticalFlow variable includes the required features for tracking the license plate in later frames. It should be noted that in the proposed implementation, we considered 10 frames for tracking, instead of tracking the vehicle in the whole scene and no huge changes in tracking accuracy were detected. Fig 11 indicates the parallel implementation of Algorithm5 in a CUDA-enabled environment. According to [40], the parallelized version of the KLT tracking algorithm can provide a large rate of speed-up due to containing multiple add, subtraction, and multiplication matrix 
processes. The definition of block and grid is the same as Algorithm2 and Algorithm4. The pixels to threads mapping is done in $O(1)$ and the row-major fetching of the pixels in each pyramid level is executed in parallel. It should be noted that the implementation codes of both CUP-based and CUDA-enabled version of Algorithms1 to 5 are presented in the Appendices section.

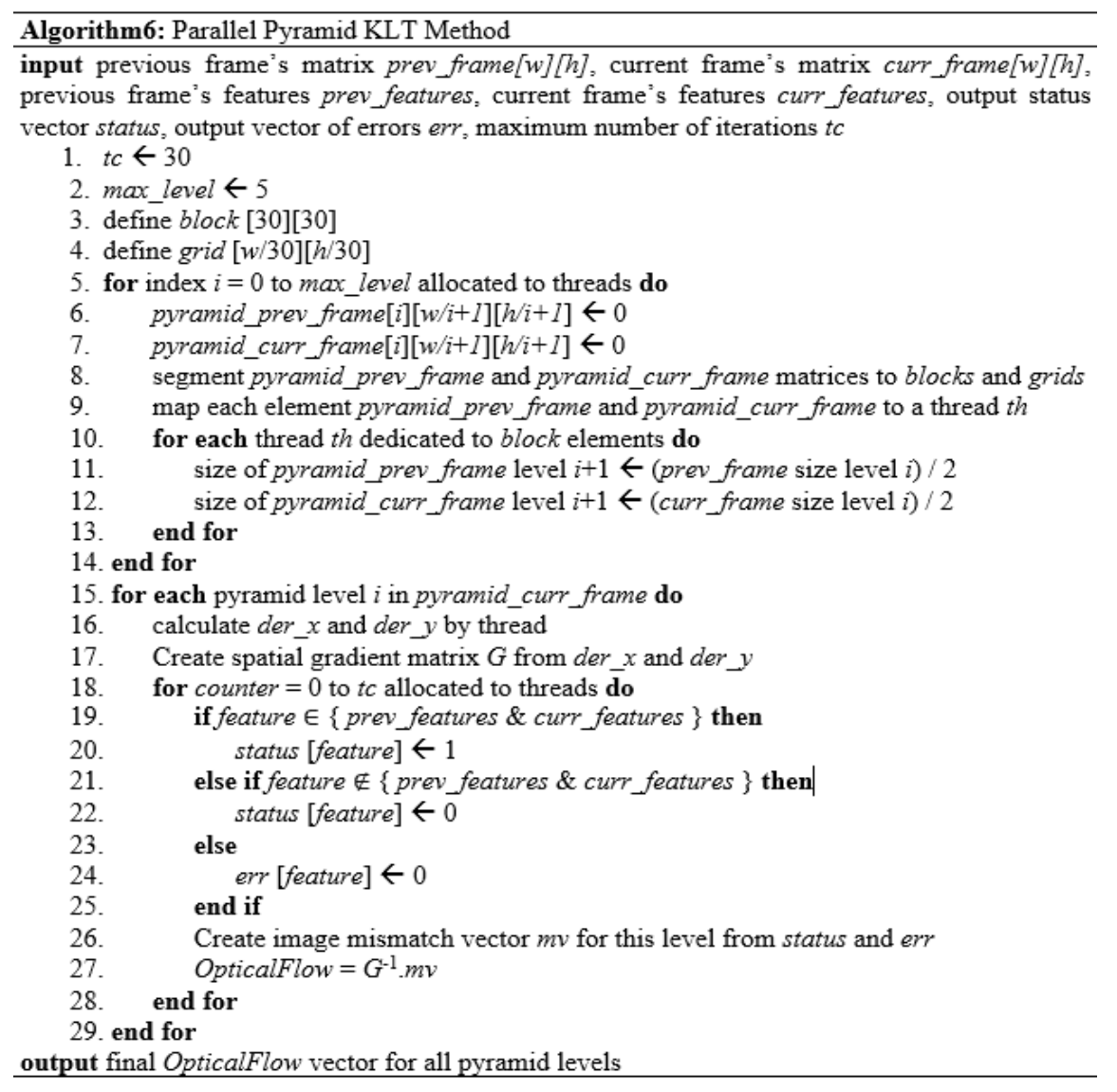

Fig.11. Parallel implementations of the pyramid KLT tracking module.

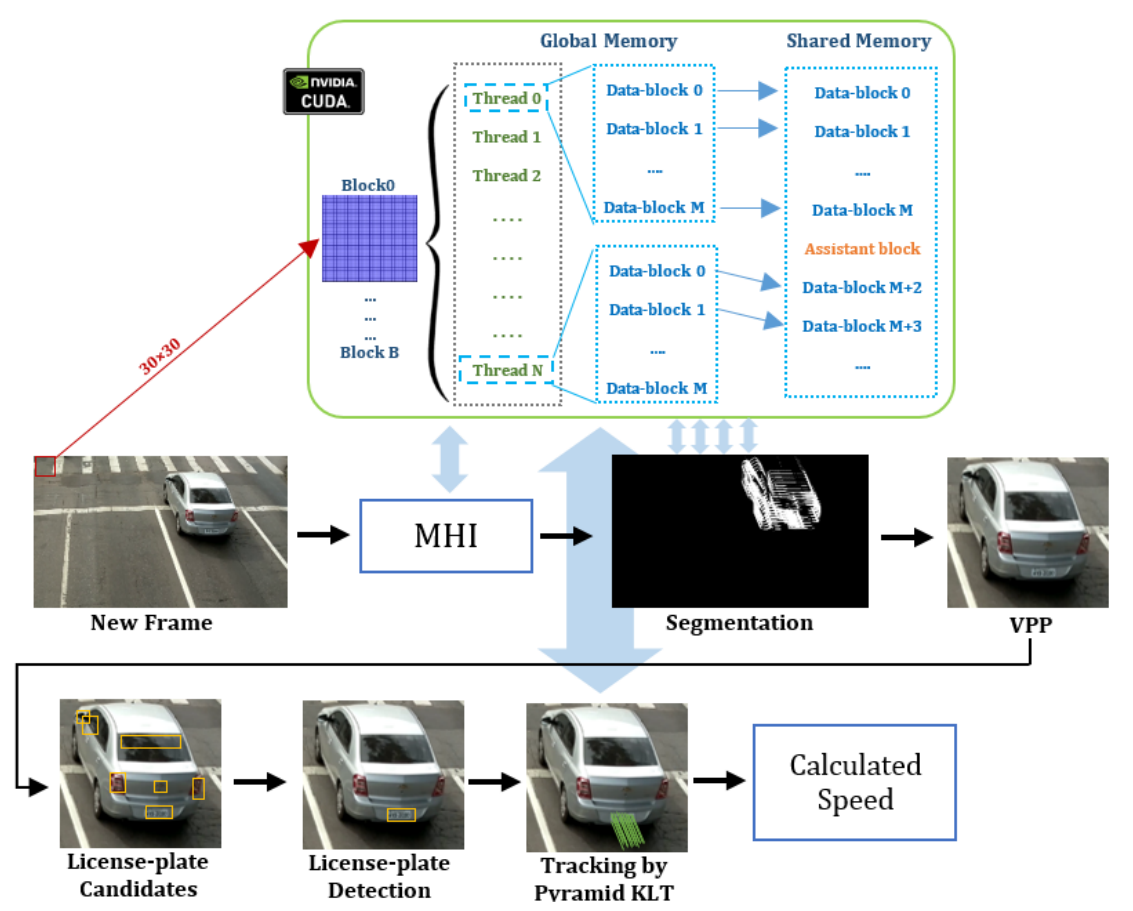

Fig.12. The overall diagram of the proposed parallelized speed measurement system. 
As a summary, Fig.12 demonstrates the overall process of the speed measurement approach and the proposed parallelized modules. As it can be seen, we have considered a $30 \times 30$ block of pixels for parallelization of MHI and segmentation modules, i.e. calculation of $H(x, y$, $t), M(x, y, t)$ matrices, respectively. On the other hand, the parallelization of the pyramid KLT was done in each level of the pyramid to track the vehicles in sequential frames. Consequently, each block contains $N+1$ threads and each thread works on $M+1$ data blocks on the global memory. The final results of the processes are transferred to the shared memory.

\section{EXPERIMENTAL RESULTS}

This section introduces the performances of the parallel implemented method presented in this article in terms of time execution. Both the parallel (GPU-enabled) and sequential (proposed in [12]) methods were analyzed on a computer with properties demonstrated in Table 1. We describe the experiment by introducing the main factors utilized for time consumption comparison.

\section{A. Dataset}

The provided dataset for the experiment is a video dataset which has been captured via a camera installed above an urban roadway. The dataset is provided by the Federal University of Technology of Paraná (FUTP) [12], including five $\mathrm{H} 264$ videos captured by a 5-megapixel CMOS image sensor with different illumination and weather conditions summarized in Table 2 . The video has been captured the rear view of vehicles, makes it suitable for license plate detection and speed measurement purposes. It has to be mentioned that due to different types of motorcycles' license plates, they have been skipped in this paper [12]. Frame resolution of the dataset is $1920 \times 1080$ pixels and the frame-rate is 30.15 frames per second. The videos are categorized into five different categories according to weather and recording conditions. Each video has a separate XML file format that contains information about that video such as vehicle speed. Table 2 shows the properties of this dataset with its corresponding speed ranges.

Table 1. Implementation Hardware And Environment

\begin{tabular}{ll} 
Hardware & Properties \\
\hline CPU & $3.5 \mathrm{GHz}$ Intel Core i7 - 7500U \\
RAM & 12 GB \\
GPU & NVIDIA GEFORCE 920MX \\
Operating System & 64-bit Windows 10
\end{tabular}

Table 2. Properties Of The Dataset.

\begin{tabular}{|c|c|c|c|c|}
\hline Dataset & Filename & \#frame & Video properties & \#Vehicles \\
\hline & Set01_video01 & 6918 & Normal illumination & 119 \\
\cline { 2 - 5 } & Set02_video01 & 12053 & High illumination & 223 \\
\cline { 2 - 5 } $\begin{array}{c}\text { The Federal } \\
\text { University of } \\
\text { Technology of } \\
\text { Paraná [12] }\end{array}$ & Set03_video01 & 24301 & $\begin{array}{c}\text { Low-light } \\
\text { illumination }\end{array}$ & 460 \\
\cline { 2 - 5 } & Set04_video01 & 19744 & $\begin{array}{c}\text { Rainy weather } \\
\text { conditions }\end{array}$ & 349 \\
\cline { 2 - 5 } & Set05_video01 & 36254 & $\begin{array}{c}\text { Extreme rain } \\
\text { weather }\end{array}$ & 869 \\
\hline Total & - & $\mathbf{9 9 2 7 0}$ & - & $\mathbf{2 0 2 0}$ \\
\hline
\end{tabular}

\section{B. Time Consumption}

To evaluate the performance of the parallelized method, we have compared it to the sequential method presented in [12]. Since there were no vast changes in the accuracy of vehicle detection, tracking and speed measurement processes in the parallelized and sequential approaches, we have only focused on the timeconsumption comparison. By comparing elapsed times for execution of the parallelized versions of $H, M$ and Pyramid KLT modules to the original, we observed a huge change in the fields of performance and efficiency. Table 3 shows the results of this evaluation in brief. It should be noted that each cell of the table refers to the average elapsed time for a particular vehicle to run the mentioned modules in different videos. As it can be seen, the parallelized versions (kernels) execute 188.87, 408.71 and 21.28 times faster than the sequential versions in calculating $H, M$ and Pyramid KLT modules, respectively.

According to Table 3, only the effect of parallelization on each module $H, M$ and Pyramid KLT are presented in terms of speed-up. In other words, Table 3 only shows the effect of parallelization on each kernel, while the whole effect of utilizing these modules in the application level is not provided. In order to review the effects of parallelization on the whole process of speed measurement, Fig.13 illustrates the average of the total time required to calculate a single vehicles' speed in various videos of the dataset. As can be seen, parallelization of the most computationally intensive modules leads to about 1.81 execution speed-up in application level in various illumination and weather conditions. To acquire these numbers, a timer triggered as a vehicle entered the ROI with a recognizable license plate and stopped as it left the region. Although the parallelized versions of $H, M$, and Pyramid KLT modules provide a robust speed-up according to Table 3 , the effect of utilizing them in the speed measurement process did not provide a vast difference, as it can be seen in Fig.13. The reason can be found in the huge amount of data transportation among GPU and CPU for processing. 
Table 3. Time Consumptions Of Sequential And Parallelized Implementations Of Modules For Each Vehicle.

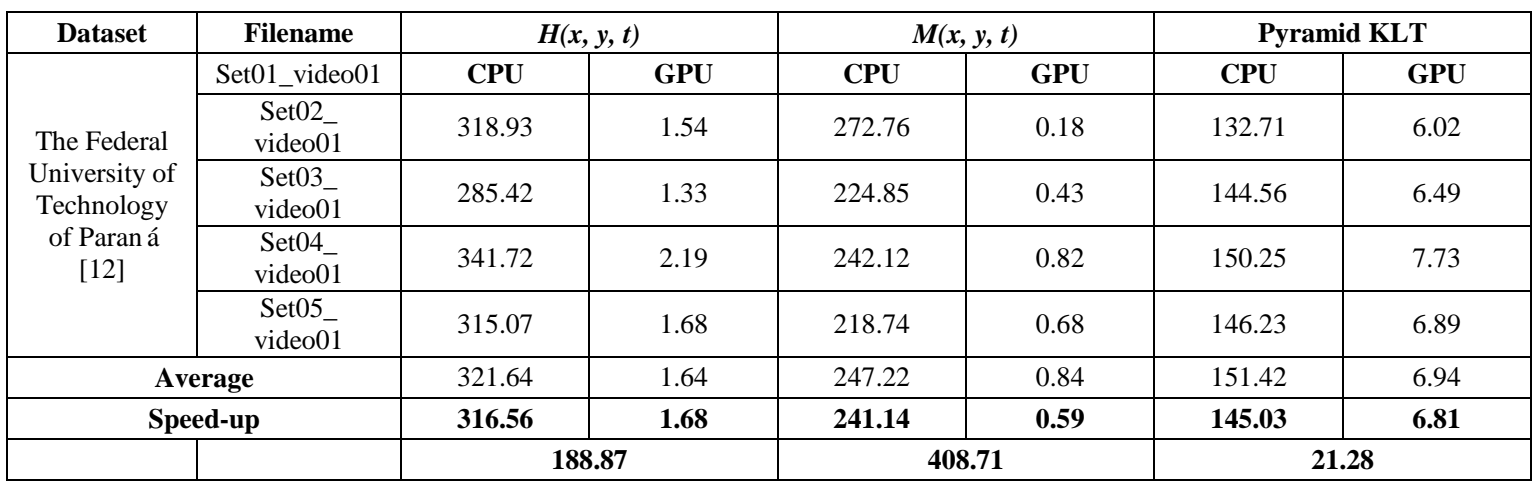

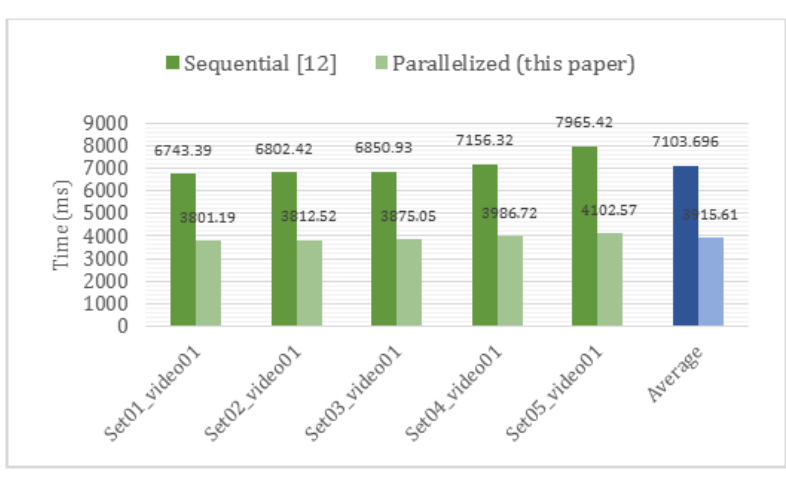

Fig.13. Total running time of sequential and CUDA-enabled implementations to calculate the speed of a single vehicle on various videos.

Finally, to provide a better demonstration of the parallelization effect on each distinct modules of the proposed speed measurement approach, Fig.14 presents the time portion required for the execution of each essential module in brief. As it can be seen, the chart shows the execution time (in milliseconds) of each distinct modules of the parallelized version in comparison with the sequential version, which has been previously presented in Fig.3. It should be noted that to acquire these execution times, the time wasted to transfer data between GPU memories and CPU are skipped. On the other hand, the effect of parallelization on the accuracy of the system compared to the sequential version was negligible.

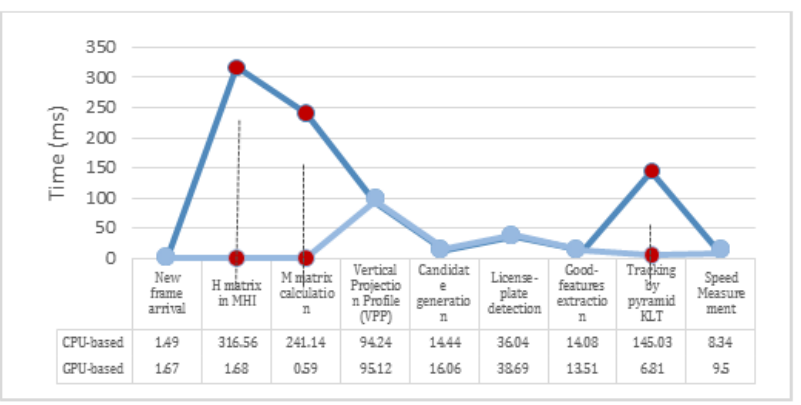

Fig.14. Profiling time portion of various modules in the system (in milliseconds).

According to Fig.14, the huge gap between $H, M$ and Pyramid KLT modules are obviously distinct (shown in red dots for both sequential and parallelized versions). As a result, the benefits and advantages presented in the experiments are adequate to make the CUDA-enabled version more applicable in almost real-time applications.

\section{CONCLUSIONS}

Intelligent Transportation Systems are used in various traffic-related applications such as roadway monitoring and vehicle counting. By utilizing cameras in ITS applications, Video-based Intelligent Transportation Systems were appeared, which can be used in various applications like speed measurement. In this paper, a parallelized version of a formerly-proposed vehicle speed measurement method is presented which has the advantage of appropriate time consumption, accuracy, and robustness. The CPU-based version of the mentioned method has three modules including vehicle detection, tracking, and speed measurement. We have realized that two functions in vehicle detection and one in the tracking phase are computationally intensive and have the potential to be highly reduced in cost. By implementing the same method in a CUDA-enabled environment and applying data-level parallelism on these modules, better throughputs and performance have been obtained. Experimental results showed that the parallelized version of the method provides 1.81 speed-up in application level to measure each vehicle's speed compared to the normal CPU-based implementation in overall. In addition, the kernel-level parallelization provided 21.28, 408.71 and 188.87 speed-up in executing three computationally intensive modules.

\section{APPENDICES}

Appendix I - Sequential and parallel implementations of $H(X, Y, T)$ module in $C++$

Below, the variable diff_mat refers to the subtraction matrix of two sequential frames. If the result of the subtraction was equal to 1, the first condition of Equation (1) is used and otherwise, the second condition would be utilized. In addition, variable current_mhi refers to the current value of MHI and prev_mhi represents its value in the previous frame. The variable mhi_duration is set to 5 in this approach, which means the system takes advantage of five frames as the history to calculate the $H$ 
matrix. In the CUDA-enabled codes, the block-size of the GPU is defined in $B X$ and $B Y$ and the resolution of the frames are defined in $D X$ and $D Y$, respectively. In addition, the function type qualifier___olobal__ refers to a kernel with the ability to be executed on the CUDA device and grid and block variables, contain the dimensions of grids and blocks. The block size is set to $30 \times 30$, which provides the best performance according to experiments. In addition,_Update_MHI_GPU is the name of kernel and row and col variables are used to choose threads. The provided pseudo-codes of these two implementations are shown in below.

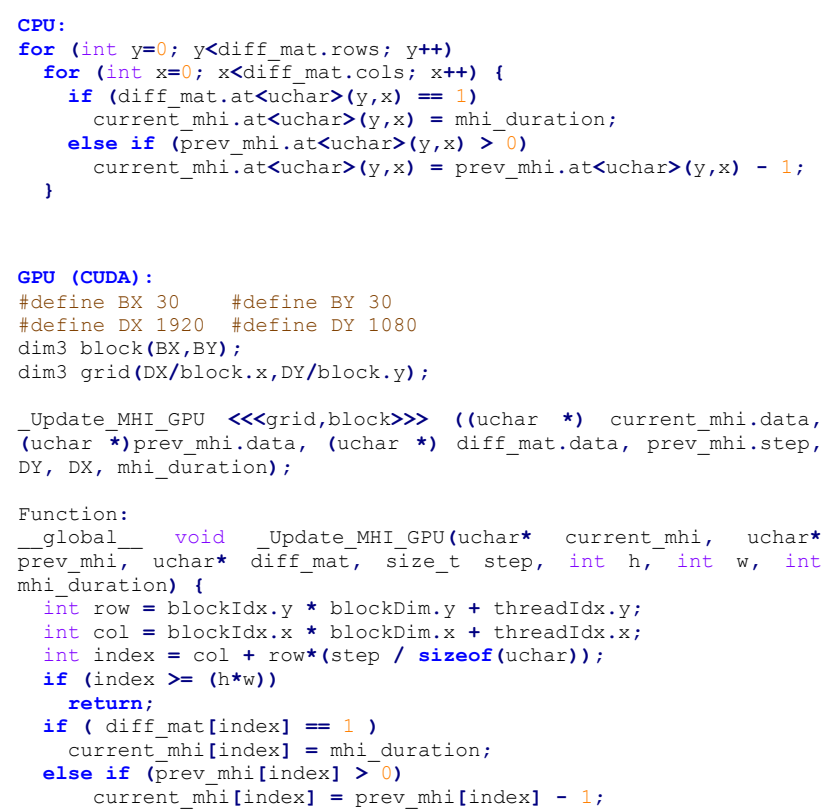

Appendix II - Sequential and parallel implementations of $M(X, Y, T)$ module in $C++$

Below, the CPU-based (sequential) and GPU-based (parallel) implementations of $M(x, y, t)$ matrix calculation method are shown, where_SegmentationBy_GPU is the name of the kernel. As it has been described before, $M(x$, $y, t)$ matrix is used for segmentation of MHI. Variable $m \_m a t$ refers to $M(x, y, t)$ matrix presented in Equation (2), thus if the value of $M H I$ was bigger than zero, the value of $M$ would be 1 and otherwise, it stores as zero. Pseudocodes of these two implementations are shown in below.

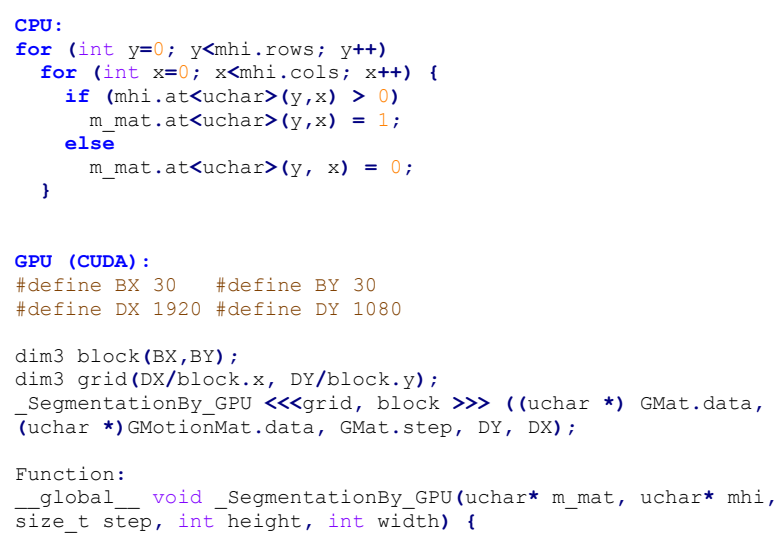

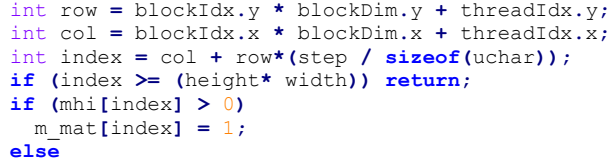

Appendix III - Sequential and parallel implementations of the pyramid KLT tracking module in $\mathrm{C}++$

Below codes illustrate the sequential and parallel implementations of the pyramid KLT module. According to these codes, prev_frame and curr_frame variables refer to the current and previous frames matrices, respectively. The candidate features of being a license plate found in the previous frame by the good features algorithm are stored in prev_features and the curr_features variable keeps the features existed in the current frame. Finally, variable $t c$ stores the terminating conditions of the search module. Pseudocodes of these two implementations are shown in below.

$\mathrm{CPU}$ :

TermCriteria tc $=$ TermCriteria (TermCriteria: $:$ COUNT +

TermCriteria: :EPS, 30, 0.01);

CalcopticalflowPyrLK (prev_frame, currfframe, prev_features, curr features, status, Size(11, 11), 5, tc, 0, 0.0001);

GPU (CUDA)

Ptr<cuda: :SparsePyrLKopticalflow> d pyrLK sparse $=$

cuda: : SparsePyrLKOpticalflow: :create (Size-(11, 11), 5, 1); d_pyrLK_sparse->calc(prev_frame, curr_frame, Prev_Points, Next_Points, d_status)

\section{REFERENCES}

[1] K.N. Qureshi and A.H. Abdullah, "A Survey on Intelligent Transportation Systems," Middle-East Journal of Scientific Research, vol. 15, No. 5, 2013.

[2] F. Zhu, Z. Li, S. Chen, and G. Xiong, "Parallel Transportation Management and Control System and its Applications in Building Smart Cities," IEEE Transactions on Intelligent Transportation Systems, vol. 17, no. 6, pp. 1576-1585, 2016.

[3] M. Bommes, A. Fazekas, T. Volkenhoff, and M. Oeser, "Video based Intelligent Transportation Systems - State of the Art and Future Development," Transportation Research Procedia, vol. 14, pp. 4495-4504, 2016.

[4] M. A. Adnan, N. Sulaiman, N. I. Zainuddin and T. B. H. T. Besar, "Vehicle Speed Measurement Technique using Various Speed Detection Instrumentation," IEEE Business Engineering and Industrial Applications Colloquium, Langkawi, pp. 668-672, 2013.

[5] Z. Marszalek, R. Sroka and T. Zeglen, "Inductive Loop for Vehicle Axle Detection from First Concepts to the System based on Changes in the Sensor Impedance Components," $20^{\text {th }}$ International Conference on Methods and Models in Automation and Robotics, Miedzyzdroje, pp. 765-769, 2015.

[6] J. Zhang, H.W. Li, L.H. Zhang and Q. Hu, "The Research Of Radar Speed Measurement System based on TMS320C6745," IEEE $11^{\text {th }}$ International Conference on Signal Processing, Beijing, pp. 1843-1846, 2012.

[7] S. Sivaraman and M. M. Trivedi, "Looking at Vehicles on the Road: A Survey of Vision-Based Vehicle Detection, Tracking, and Behavior Analysis, " IEEE Transactions on Intelligent Transportation Systems, vol. 14, no. 4, pp. 1773-1795, 2013.

[8] R. Minetto, N. Thome, M. Cord, J. Stolfi, and N. J. Leite, "T-HOG: An Effective Gradient-Based Descriptor for 
Single Line Text Regions, " Pattern Recognition Elsevier, vol. 46, no. 3, pp. 1078-1090, 2013.

[9] J. Shi, and C. Tomasi, "Good Features to Track," IEEE International Conference on Computer Vision and Pattern Recognition, pp. 593-600, Seattle, 1994.

[10] J. Y. Bouguet, "Pyramidal Implementation of the Lucas Kanade Feature Tracker," Intel Corporation, Microprocessor Research Labs, Technical Report, 2000.

[11] D. De Donno, A. Esposito, L. Tarricone and L. Catarinucci, "Introduction to GPU Computing and CUDA Programming: A Case Study on FDTD, " IEEE Antennas and Propagation Magazine, vol. 52, no.3, June 2010.

[12] D. C. Luvizon, B. T. Nassu, and R. Minetto, "A VideoBased System for Vehicle Speed Measurement in Urban Roadways," IEEE Transactions on Intelligent Transportation Systems, vol. 18, no. 6, pp. 1393-1404, 2017.

[13] D. Dailey, F. Cathey, and S. Pumrin, "An Algorithm to Estimate Mean Traffic Speed using Uncalibrated Cameras," IEEE Transactions on Intelligent Transportation Systems, vol. 1, no. 2, pp. 98-107, 2000.

[14] V. Madasu and M. Hanmandlu, "Estimation of Vehicle Speed by Motion Tracking on Image Sequences," IEEE Intelligent Vehicles Symposium, pp. 185-190, 2010.

[15] S. Dogan, M. S. Temiz, and S. Kulur, "Real-time Speed Estimation of Moving Vehicles from Side View Images from an Uncalibrated Video Camera," Sensors, vol. 10, no. 5, pp. 4805-4824, 2010.

[16] C. H. Xiao and N. H. C. Yung, "A novel Algorithm for Estimating Vehicle Speed from Two Consecutive Images, " IEEE Workshop on Applications of Computer Vision, p. 12-13, 2007.

[17] H. Zhiwei, L. Yuanyuan, and Y. Xueyi, "Models of Vehicle Speeds Measurement with a Single Camera," International Conference on Computational Intelligence and Security, pp. 283-286, Harbin, 2007.

[18] C. Maduro, K. Batista, P. Peixoto, and J. Batista, "Estimation of Vehicle Velocity and Traffic Intensity Using Rectified Images, ” IEEE International Conference on Image Processing, pp. 777-780, San Diego, 2008.

[19] H. Palaio, C. Maduro, K. Batista, and J. Batista, "Ground Plane Velocity Estimation Embedding Rectification on a Particle Filter Multitarget Tracking," IEEE International Conference on Robotics and Automation, pp. 825-830, Kobe, 2009.

[20] L. Grammatikopoulos, G. Karras, and E. Petsa, "Automatic Estimation of Vehicle Speed from Uncalibrated Video Sequences," Modern Technologies, Education and Professional Practice in Geodesy and Related Fields, pp. 332-338, 2005.

[21] T. Schoepflin and D. Dailey, "Dynamic Camera Calibration of Roadside Traffic Management Cameras for Vehicle Speed Estimation," IEEE Transactions on Intelligent Transportation Systems, vol. 4, no. 2, pp. 9098, 2003.

[22] G. Garibotto, P. Castello, E. Del Ninno, P. Pedrazzi, and G. Zan, "Speedvision: Speed Measurement by License Plate Reading and Tracking," IEEE Transactions on Intelligent Transportation System, pp. 585-590, Oakland, 2001.

[23] W. Czajewski and M. Iwanowski, "Vision-based Vehicle Speed Measurement Method," International Conference on Computer Vision and Graphics, vol. 10, pp. 308-315, Berlin, 2010.

[24] M. Garg and S. Goel, "Real-time License Plate Recognition and Speed Estimation from Video Sequences,"
ITSI Transactions on Electrical and Electronics Engineering, vol. 1, no. 5, pp. 1-4, 2013.

[25] C. N. E. Anagnostopoulos, I. E. Anagnostopoulos, I. D. Psoroulas, V. Loumos, and E. Kayafas, "License Plate Recognition from Still Images and Video Sequences: A survey," IEEE Transactions on Intelligent Transportation Systems, vol. 9, no. 3, pp. 377-391, 2008.

[26] S. Du, M. Ibrahim, M. Shehata, and W. Badawy, "Automatic License Plate Recognition (ALPR): A Stateof-the-art Review," IEEE Transactions on Circuits Systems and Video Technology, vol. 23, no. 2, pp. 311325, 2013.

[27] B. Li, B. Tian, Y. Li, and D. Wen, "Component-based License Plate Detection using Conditional Random Field Model, " IEEE Transactions on Intelligent Transportation Systems, vol. 14, no. 4, pp. 1690-1699, 2013.

[28] B. D. Lucas and T. Kanade, "An Iterative Image Registration Technique with an Application to Stereo Vision," Joint Conference on Artificial Intelligence, pp. 674-679, 1981.

[29] A. Bobick and J. Davis, "The Recognition of Human Movement using Temporal Templates," IEEE Transactions on Pattern Analysis and Machine Intelligence, vol. 23, no. 3, pp. 257-267, 2001.

[30] J. Ha, R. Haralick, and I. Phillips, "Document Page Decomposition by the Bounding-Box Project," International Conference on Document Analysis and Recognition, vol. 2, pp. 1119-1122, Montreal, 1995.

[31] T. Retornaz and B. Marcotegui, "Scene Text Localization based on the Ultimate Opening," International Symposium on Mathematical Morphology, vol. 1, pp. 177-188, 2007.

[32] T. H. Cormen, C. E. Leiserson, R. L. Rivest, and C. Stein, "Introduction to Algorithms," $3^{\text {rd }}$ Edition, ISBN: 0262033844, The MIT Press, 2009.

[33] R. Minetto, N. Thome, M. Cord, N. J. Leite, and J. Stolfi, "SnooperText: A Text Detection System for Automatic Indexing of Urban Scenes," Computer Vision and Image Understanding Elsevier, vol. 122, pp. 92-104, 2014.

[34] G. Wang, Z. Hu, F. Wu, and H. T. Tsui, "Single View Metrology from Scene Constraints," Elsevier Image and Vision Computing, vol. 23, no. 9, pp. 831-840, 2005.

[35] D. Zheng, Y. Zhao, and J. Wang, "An Efficient Method of License Plate Location," Pattern Recognition Letters Elsevier, vol. 26, no. 15, pp. 2431-2438, 2005.

[36] B. Epshtein, E. Ofek, and Y. Wexler, "Detecting Text in Natural Scenes with Stroke Width Transform," IEEE International Conference on Computer Vision and Pattern Recognition, pp. 886-893, San Francisco, 2010.

[37] D. G. R. Bradski and A. Kaehler, "Learning OpenCV: Computer Vision with the OpenCV Library," $1^{\text {st }}$ Edition, ISBN: 0596516134, O’Reilly Media, 2008.

[38] H. Li, M. Feng, and X. Wang, "Inverse Perspective Mapping based Urban Road Markings Detection," IEEE International Conference on Cloud Computing and Intelligent Systems, vol. 03, pp. 1178-1182, Hangzhou, 2012.

[39] P. KaewTraKulPong and R. Bowden, "An Improved Adaptive Background Mixture Model for Real-time Tracking with Shadow Detection," $2^{\text {nd }}$ European Workshop on Advanced Video-based Surveillance Systems, Genova, 2002.

[40] M. Chonglei, J. Hai and J. Jeff, "CUDA-based AES Parallelization with Fine-tuned GPU Memory Utilization, " IEEE International Symposium on Parallel and Distributed Processing, 2010.

[41] J. Hedborg, J. Skoglund and M. Felsberg, “KLT Tracking 
Implementation on the GPU," Swedish Symposium in Image Analysis, 2007.

\section{Authors' Profiles}

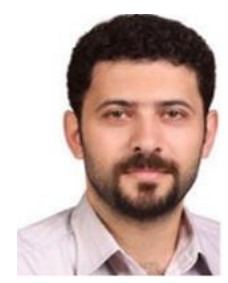

Abdorreza Joe Afshany received his BSc degree in computer software engineering in 2010 and his MSc degree in software engineering at the University of Guilan in 2018. His main research interests include motion detection, image processing, video processing and applying computer vision techniques to practical problems.

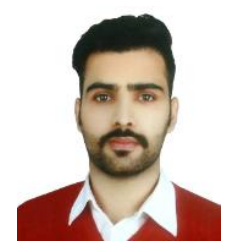

Ali Tourani is a research software engineer in the University of Guilan Business Incubar and Guilan Science and Technology Park. He received his $\mathrm{BSc}$ degree in computer software engineering in 2013 and his MSc degree in software engineering in 2019 from the University of Guilan. He is also the Chief of Guilan University Student Branch of Iran Society of Machine Vision and Image Processing. His main research interests include motion detection and tracking, machine vision, Digital Image Processing and video-based Intelligent Transportation Systems.

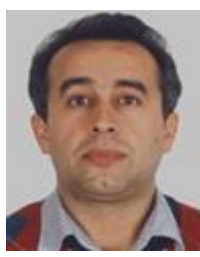

Asadollah Shahbahrami received the BSc and MSc degrees in computer engineering (hardware and machine intelligence) from Iran University of Science and Technology and Shiraz University in 1993 and 1996, respectively. He was offered a faculty position in the Department of Electrical Engineering at University of Guilan. He has been working at University of Guilan since August 1996. In January 2004, he joined the Faculty of Electrical Engineering, Mathematics, and Computer Science, Delft University of Technology, Delft, The Netherlands, as a full-time Ph.D. student under advisors Prof. Stamatis Vassiliadis and Dr. Ben Juurlink. He received his PhD degree in September 2008 from Delft University of Technology. $\mathrm{He}$ has an assistant professor position in Department of Computer Engineering at the University of Guilan. His research interests include advanced computer architecture, image and video processing, multimedia instructions set design, reconfigurable computing, parallel processing, and SIMD programming.



Saeed Khazaee was a full-time faculty member at Azad University and a visiting lecturer at the University of Guilan, Iran. He received a full scholarship from Azad university for his M.Sc. program. He has also been awarded several times related to his research. He has been in contact with several universities and companies to increase his research productivity. Saeed currently is a Ph.D. Candidate at the Centre for Pattern Recognition and Machine Intelligence, Concordia University, Montreal. Saeed does research in Computer and Society, Data mining, Image Processing and Pattern Recognition. He has published 11 journal or conference papers, and 1 book chapter in the field of Data mining, pattern recognition, and image processing. He was also a reviewer for several conferences in Iran and Canada. He is receiving a full scholarship from CENPARMI and "Concordia International Tuition Award of Excellence". He was also awarded by "Concordia University Conference and Exposition Award".

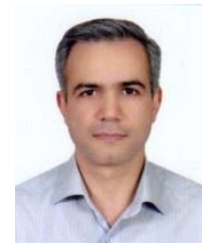

Alireza Akoushideh received the B.Sc. and M.Sc. degree in Electrical engineering from University of Guilan and Amirkabir University of Technology (Tehran Polytechnic) in 1997 and 2000, respectively. From 2001 until now, he is a faculty member of Technical and Vocational University, Shahid-Chamran community college, Rasht, Iran. He got his Ph.D. degree from Shahid-Beheshti University, Tehran, Iran in 2016. As a visiting researcher, he worked with the SCS group in the Twente University, the Netherlands from January to September 2015. $\mathrm{He}$ has taught courses in FPGA, microprocessor and microcontrollers, computer architecture, and digital circuits. His research interests include machine vision, texture analysis, FPGA implementation, and parallel processing.

How to cite this paper: Abdorreza Joe Afshany, Ali Tourani, Asadollah Shahbahrami, Saeed Khazaee, Alireza Akoushideh, "Parallel Implementation of a Video-based Vehicle Speed Measurement System for Municipal Roadways", International Journal of Intelligent Systems and Applications(IJISA), Vol.11, No.11, pp.25-37, 2019. DOI: 10.5815/ijisa.2019.11.03 